\title{
Linha de cuidado para crianças e adolescentes vivendo com HIV: pesquisa participante com profissionais e gestores ${ }^{a}$
}

\author{
Line of care for children and adolescents living with HIV: participatory research with professionals and
} managers

Línea de atención para niños y adolescentes con VIH: investigación participativa con profesionales y gerentes

Daniela Dal Forno Kinalski ${ }^{1}$ (B)

Raquel Einloft Kleinubing ${ }^{1}$ (C)

Marília Alessandra Bick ${ }^{1}$ (D)

Tassiane Ferreira Langendorf ${ }^{2}$ (D)

Stela Maris de Mello Padoin² (B)

Cristiane Cardoso de Paula² (D)

1. Universidade Federal de Santa Maria, Programa de Pós-Graduação de Enfermagem. Santa Maria, RS, Brasil.

2. Universidade Federal de Santa Maria,

Departamento de Enfermagem. Santa Maria, RS, Brasil.

\section{RESUMO}

Objetivo: Construir uma linha de cuidado para a atenção à saúde às crianças e adolescentes com HIV. Método: Pesquisa participante desenvolvida por meio da técnica de grupo focal com 23 profissionais e gestores da atenção primária e especializada, em município do Sul do Brasil. O material empírico foi submetido à análise de conteúdo temática. Resultados: $\mathrm{O}$ fato de a atenção à saúde para esta população ser realizada, majoritariamente, nos serviços especializados fomentou a discussão de aspectos conceituais, estruturais e sociais acerca da temática. Pactuou-se como deve ser realizada a atenção centrada no usuário e compartilhada entre os serviços, considerando os atributos qualificadores da atenção. As atribuições de cada serviço, as articulações e a operacionalização foram definidas para a integração entre os pontos da rede. A validação das ideias centrais culminou com a construção da linha de cuidado para crianças e adolescentes vivendo com HIV. Conclusão e implicações para a prática: A implantação desse produto adaptado ao cotidiano assistencial dos municípios requer ações estratégicas para a integração dos pontos de atenção e para qualificação da atenção à saúde dessa população.

Palavras-chave: Assistência Integral à Saúde; Saúde da Criança; Saúde do Adolescente; HIV; Síndrome da Imunodeficiência Adquirida.

\begin{abstract}
Objective: To build a network for the health care of children and adolescents living with HIV. Method: Participatory research developed through the focus group technique with 23 professionals and managers of the primary and specialized care services, in a city in the south of Brazil. The empirical data was subjected to thematic content analysis. Results: The fact that health care provided to this population is mostly performed in specialized services fostered the discussion about the conceptual, structural and social aspects of the theme. It was agreed that the assistance should be performed centered on the user and shared among the services, considering the qualifying attributes of care. The assignments of each service, the articulations and the operationalization were defined for integration among the network points. The validation of the main ideas culminated in the construction of a care network for children and adolescents living with HIV. Conclusion and implications for practice: The implementation of this product, adapted to the municipalities' daily assistance practice, requires strategic actions for integrating the points of care and for qualifying health care for this population.
\end{abstract}

Keywords: Comprehensive Health Care. Child Health. Adolescent Health. HIV. Acquired immunodeficiency syndrome.

\section{RESUMEN}

Objetivo: Construir una línea de cuidado para la atención a la salud de niños y adolecentes con VIH. Método: Investigación participativa desarrollada a través de la técnica de grupos focales con 23 profesionales y gestores de la atención primaria y especializada, en un municipio del Sur del Brasil. El material empírico se sometió a un análisis de contenido temático. Resultados: El hecho de que la atención de la salud de esta población realizarse principalmente en servicios especializados, fomentó la discusión sobre los aspectos conceptuales, estructurales y sociales del tema. Se ha definido como debe realizarse la atención centrada en el usuario y compartida entre los servicios, considerando los atributos calificativos de la atención. Los atributos de cada servicio, las articulaciones y las operacionalizaciones se definieron para la integración entre los puntos de la red. La validación de las ideas centrales culminó con la construcción de la línea de cuidado para niños y adolescentes que viven con VIH. Conclusión e implicaciones para la práctica: La implantación de este producto, adaptado a la asistencia diaria de los municipios exige acciones estratégicas para la integración de los puntos de atención y para la calificación de la atención sanitaria de esta población.

Palabras clave: Atención Integral de Salud; Salud de los niños; Salud del adolescente; VIH; Síndrome de Inmunodeficiencia Adquirida.
Autor correspondente:

Cristiane Cardoso de Paula.

E-mail: cristiane.paula@ufsm.br.

Recebido em 10/07/2020.

Aprovado em 02/09/2020.

DOI:https://doi.org/10.1590/2177-9465-EAN-2020-0266 


\section{INTRODUÇÃO}

O relatório de resposta à Agenda 2030, no que se refere ao terceiro Objetivo de Desenvolvimento Sustentável, mostra progresso na melhoria da saúde de milhões de pessoas, na redução da mortalidade materna e infantil e no combate às doenças transmissíveis. ${ }^{1}$ Contudo, dentre as metas universais de controle de infecção pelo vírus da imunodeficiência humana (HIV), uma aponta o declínio aquém do necessário para cumprir as metas da Estratégia Global do Programa Conjunto das Nações Unidas sobre o HIV/AIDS (UNAIDS) para 2016-2021.2

O Plano de Ação do UNAIDS inclui as seguintes linhas estratégicas: o acesso ampliado e equitativo aos serviços para controle da infecção pelo HIV, com integralidade e qualidade. Garantir esse acesso ampliado exige adaptar o modelo de prestação de serviços às necessidades das populações prioritárias vulneráveis com base nas condições locais da epidemia. Ainda no que se refere à prestação de serviço, deve-se adaptá-la com base nas abordagens centradas na pessoa, na comunidade e em redes integradas de serviço de saúde, tendo em vista a melhora da saúde materna e infantil. ${ }^{3}$

Para o cumprimento de tais metas e do plano de ação, o Sistema Único de Saúde (SUS) do Brasil, apoiado no modelo de Redes de Atenção à Saúde (RAS), propôs a descentralização do acompanhamento clínico da Síndrome da Imunodeficiência Adquirida dos serviços especializados. ${ }^{4,5} \mathrm{O}$ processo de descentralização é potencializado pela corresponsabilidade e não pela transferência de responsabilidade ${ }^{6}$ e demanda a integração das ações ofertadas nos serviços (horizontal) e entre os serviços (vertical). Esse processo, além de ser uma emergência é um desafio para a organização dos serviços e funcionamento das equipes. ${ }^{7}$

Para abordar a saúde materno-infantil, mais especificamente a saúde da criança e a saúde do adolescente, é essencial reconhecer a relevância da corresponsabilização entre os serviços para testagem anti-HIV no pré-natal e profilaxia em tempo oportuno para diminuir a incidência de transmissão vertical. Também, é indispensável acompanhar o tratamento precoce das crianças infectadas e a adesão ao tratamento para que sua efetividade aumente a expectativa de vida, caracterizando a condição crônica dessa infecção. Logo, a população de crianças e adolescentes infectados pelo HIV, que necessita de acompanhamento de crescimento e desenvolvimento, ampliou a solicitação de rotina permanente de acompanhamento clínico, laboratorial e de adesão ao tratamento medicamentoso, que acontece, majoritariamente, em serviços especializados. ${ }^{8}$

Essa integração entre os serviços torna-se uma estratégia para qualificar a atenção à saúde ${ }^{9}$ e pode ser estabelecida por meio de um fluxo das crianças no sistema de saúde. É fundamental o fortalecimento da capacidade de comunicação entre os serviços e a definição de atribuições de cada serviço (ponto da RAS) para garantir o acesso, atender com integralidade e longitudinalidade as demandas dessa população e coordenar os encaminhamentos. ${ }^{10}$ Este estudo teve como objetivo construir uma linha de cuidado para a atenção à saúde de crianças e adolescentes vivendo com HIV.

\section{MÉTODO}

Estudo participante ${ }^{11}$ mediado pela técnica de Grupo Focal (GF). ${ }^{12}$ Essa técnica proporcionou a discussão da opinião dos participantes, a troca de experiências acerca do atendimento de crianças e adolescentes vivendo com HIV e a elaboração de estratégias para a solução dos problemas da prática elencados pelo grupo nas sessões.

Foram cenários para coleta de dados os serviços de Atenção Primária à Saúde (APS), 18 Unidades Básicas de Saúde (UBS) e 13 Estratégias de Saúde da Família (ESF) e 2 especializados (municipal e federal) em município da região central do Rio Grande do Sul. Na etapa de recrutamento dos participantes, foi enviado um convite formal para os serviços e alguns foram visitados para definir a melhor estratégia para o recrutamento. Foi criada uma lista (nome/contato) daqueles profissionais interessados e que atendessem ao critério de inclusão: pertencer ao quadro efetivo e não estar afastado do trabalho. Não houve delimitação de tempo de atuação e/ou categoria profissional.

A etapa de campo foi desenvolvida em quatro sessões grupais, com agendamento de horário vespertino, local central, com estrutura adequada, no período de maio a junho de 2015. As sessões foram conduzidas por uma moderadora e duas observadoras com experiência na técnica de GF e no tema HIV, tiveram duração média de duas horas e foram gravadas. $\mathrm{O}$ debate foi guiado por questões relacionadas ao objetivo de cada sessão: Quais ações são desenvolvidas no seu serviço para a atenção à saúde de crianças e adolescentes vivendo com HIV? E quais ações poderiam ser desenvolvidas no seu serviço? O que é de responsabilidade do meu serviço de saúde para atender a esta população? Como pode ser pensada a atenção a esta população no município? A validação das ideias centrais aconteceu a partir da apresentação da síntese de todas as sessões para os participantes e foi centrada na linha de cuidado (LC) para crianças e adolescentes vivendo com HIV, produto da construção coletiva do GF.

O banco de dados foi constituído pelas transcrições e registros dos diários de campo submetidos à análise de conteúdo. ${ }^{13}$ Esse projeto foi aprovado pelo Comitê de Ética em Pesquisa (parecer no 924.646/2014). Para apresentação dos resultados, foram utilizadas codificações: P: Participantes; ESF, UBS ou SE: indicação do serviço; e GF-S1: indicação da sessão do GF.

\section{RESULTADOS}

Os participantes foram 23 profissionais, sendo 11 da ESF, quatro da UBS, dois do Serviço especializado municipal, quatro do Serviço especializado federal e dois gestores pertencentes à Secretaria Municipal de Saúde (SMS) que possuíam cargos de coordenação da APS e da Política de HIV/aids. A maioria dos profissionais possuía até cinco anos de atuação no referido serviço, e seu nível de ensino era Graduação e Pós-Graduação. 
Esses dados foram coletados a partir da apresentação pessoal de cada profissional durante as sessões no GF.

Na discussão grupal, os participantes compreenderam que a organização da atenção à saúde dessa população perpassa os elementos: conceitual, de estrutura e social (Quadro 1). Os elementos foram sintetizados a partir da análise de conteúdo dos registros das falas nos GF.

Entre os elementos conceituais de atenção, foi foco de discussão pelos participantes o entendimento de que essa infecção passou a ser considerada como uma condição crônica de saúde que pode ser acompanhada no serviço de APS.

[...] É que hoje a gente tem que entender que o HIV é uma doença crônica como qualquer outra. E que antes a gente também não queria o diabético na APS [...] a gente sabe que é nosso esse paciente (com HIV), nós precisamos acolher, nós precisamos tratar, nós precisamos acompanhar [...] [P7/SE GF-S3]

Para os participantes, devido à condição crônica complexa, a atenção à saúde das crianças e dos adolescentes vivendo com HIV está concentrada nos serviços especializados ou em serviços hospitalares. Isso culmina na prática centralizada dos profissionais, ao encontro do que almejam os usuários. Os participantes discutiram o quanto as crianças e adolescentes infectados precisam ser acompanhados pelo especialista em infectologia, revelando outro elemento conceitual: a descentralização da atenção à saúde do serviço especializado.

[...] A maior parte do atendimento delas é prestado no serviço especializado e não na APS [P15/ESF GF-S1].

[...] a gente faz testagem e aconselhamento e todos os pacientes vêm para o cuidado no serviço especializado [P20/SS GF-S2].
[...] Quando as crianças chegavam com a carteirinha escrito: "continuar vacinando no serviço especializado", eles assumiram e nós não questionamos [P10/ESF GF-S2].

[...] seria interessante que as pessoas com HIV tivessem como referências as unidades básicas de saúde, e ela (usuária) me disse: o que o médico do postinho tem a me oferecer? Eu preciso de um infectologista [P8/ESF GF-S1].

[...] Realmente, o adolescente é tudo com o serviço especializado [P21/SE GF-S1].

Outro resultado dessa pesquisa foi o reconhecimento do elemento corresponsabilidade entre os serviços de APS e o serviço especializado pelos profissionais. Isso indica a necessidade de estabelecer mudanças na organização desses serviços.

[...] parece que os pacientes HIV são propriedades do serviço especializado, principalmente as crianças. Isso formou uma cultura que a gente precisa mudar [P8/ESF GF-S1].

[...] Ela [médica da ESF] me disse que nunca atendeu nenhuma criança com HIV, ou seja, egressos lá do serviço especializado que são ou deveriam ser atendidos também nas unidades básicas de saúde [...] [P1/SE GF-S1].

Este estudo aponta a existência de barreiras para o desenvolvimento de tal corresponsabilização, o que envolve o conceito de territorialização e adscrição dos usuários. Esse resultado está presente no relato dos profissionais da APS que revelaram desconhecer o registro de crianças e adolescentes com HIV adscritas no território de seu serviço de saúde.

[...] Na nossa área, a gente acha que tem, mas não tem conhecimento de quem são essas pessoas [com HIV] em nosso território [P8/ESF GF-S1].

Quadro 1. Quadro sinóptico da discussão com profissionais e gestores acerca da atenção à saúde de crianças e adolescentes vivendo com HIV. Brasil, 2015.

\section{ELEMENTOS \\ CONCEITUAL}

Condição crônica de saúde da pessoa com HIV;

Descentralização da atenção à saúde do serviço especializado;

Corresponsabilidade da APS e serviço especializado neste atendimento;

Territorialização e adscrição da população específica ao serviço de APS.

DE ESTRUTURA

Gestão Física Pessoal

Superlotação;

Demanda de atenção de âmbito local e regional; Limitada.

Escassez de profissionais especialistas;

Transferências entre os serviços. Falta de capacitação no tema.

SOCIAL

Estigma; Preconceito; Ética profissional.

Fonte: Grupo Focal, elaboração própria. 
[...] Eu não tenho como dizer, bem sinceramente, quem são as crianças [com HIV] nessa ESF [P17/ESF GF-S1].

No que tange à análise do elemento estrutura, emergiram informações oriundas de gestão, física e de pessoal. Como elemento estrutural de gestão, tem-se a superlotação do serviço especializado e a atenção de âmbito local e regional. Isso, devido ao acolhimento de outros municípios e de outras Coordenadorias de Saúde da região.

[...] recebemos pacientes que vêm de outros municípios, não só a 4a coordenadoria, e temos que atender [P1/ SE GF-S1].

Os profissionais relataram dificuldades quanto à transferência dos usuários entre o serviço especializado e de APS, inclusive pela inexistência de um prontuário único e de um sistema informatizado de comunicação. Na população de crianças, os profissionais reconhecem o desafio de transferência do serviço de infectologia pediátrico para o de adultos.

[...] Se tu envia a criança para outro serviço, tu não sabe se ela foi, se ela chegou e qual foi a resposta do atendimento [P1/SE GF-S2].

[...] E, muitas vezes, a gente não tem nem a informação de que o paciente é HIV, porque não é informatizado, não tem a referência do serviço que é utilizado, não sabe se o paciente está estável, então precisa inclusive pedir os exames [P2/ESF GF-S2].

[...] o serviço especializado consegue de certa forma contemplar o atendimento pediátrico [...] adolescentes estão virando adultos, e tem problema de marcação de consultas, a agenda vai até os 20 anos [...] seria muito bom termos um médico responsável pelo atendimento nessa transição [...] talvez seja a hora de transferi-lo para a rede $[\mathrm{P} 1 / \mathrm{SE}$ GF-S1].

A questão da estrutura física diz respeito à limitação de espaço no serviço, e a de pessoal se refere, ainda, à escassez de profissionais infectologistas pediatras e profissionais das demais especialidades no quadro efetivo na APS, além da falta de capacitação no tema para a atenção à população específica.

[...] o limite estrutural, inclusive, não possibilita ampliar o serviço [P21/SE GF-S1].

[...] as maiores dificuldades são de estrutura física e de profissional [...] [P20/SE GF-S1].

[...] tudo chega para eles [profissionais da APS] e tem que ser absorvido por uma equipe mínima de um clínico, um enfermeiro e um técnico [...] [P7/SE GF-S2]

[...] São somente dezessete infectologistas pediatras para o estado inteiro [P1/SE GF-S1].
[...] a maioria de profissionais da ponta precisa de capacitação [P7/SE GF-S2].

Além disso, o preconceito tanto dos usuários quanto dos profissionais e a ausência de ética entre profissionais que não respeitam o direito ao sigilo representaram, para os participantes, barreiras para o acesso e para o vínculo com o serviço de APS.

[...] Eles não querem revelar que são HIV, eles têm medo de ser discriminados na sua comunidade [P8/ESF GF-S1].

[...] O pessoal da rede básica tem essa noção de que eles [familiares das crianças] não querem ser atendidos na rede básica, não é só porque não têm confiança no profissional, eles têm medo do preconceito [P7/SE GF-S2].

[...] Alguns serviços acolhem bem e outros não, por vários motivos, inclusive, o preconceito do próprio profissional da saúde [P20/SE GF-S2].

[...] o maior problema do preconceito é de que ele reverte na não adesão ao tratamento [P1/SE GF-S3].

Os participantes indicaram a discussão das atribuições dos serviços na constituição de uma RAS e a articulação e operacionalização da atenção à saúde (Quadro 2). Enfim, a partir da síntese das ideias centrais e com a validação conjunta dessas ideias no GF, os participantes expressaram a concordância entre a síntese e as ideias discutidas. Assim, houve a proposição da LC para crianças e adolescentes vivendo com HIV.

Pactuou-se como deve ser realizada a atenção centrada no usuário e compartilhada entre os serviços na perspectiva de construção de RAS, considerando a qualidade da atenção a partir dos atributos acesso, longitudinalidade, integralidade $e$ coordenação. Assim, os profissionais definiram os nós entre os pontos da RAS. O primeiro nó consistiria na definição de atribuições entre os níveis de atenção, buscando o compartilhamento do cuidado, como forma de garantir uma resposta mais integral à epidemia. Quanto aos serviços especializados: oferecer diagnóstico, aconselhamento, avaliação e tratamento. A APS foi definida como a responsável pelo acolhimento dos usuários, exercendo seu papel de responsável e coordenadora do cuidado e desenvolvendo a atenção por meio da prevenção, promoção e educação em saúde. Isso levou à escolha da estratificação de risco como uma estratégia inicial para o cuidado compartilhado. Tal estratificação considerava os níveis de CD4, que é o grupamento de diferenciação 4 (cluster of differentiation), e de CV, que são marcadores biológicos para análise do quadro clínico da infecção.

O segundo nó consistiu na articulação entre os serviços, organizando a demanda conforme a idade. $O$ terceiro nó foi composto pela operacionalização de ações: transferências dos usuários entre os pontos, contato telefônico para a coordenação do cuidado, busca ativa e monitorização da população adscrita, registro dos usuários nos serviços, profissional de referência nos serviços e fluxo na rede. Por fim, os participantes do GF ilustraram a LC (Figura 1). 
Quadro 2. Atribuições de cada serviço na constituição da Rede de Atenção à Saúde de crianças e adolescentes com HIV. Brasil, 2015.

\begin{tabular}{|c|c|c|}
\hline \multicolumn{3}{|c|}{ ATRIBUIÇÃO } \\
\hline \multicolumn{3}{|c|}{ Estabelecimento de funções (competências) entre os pontos de atenção. } \\
\hline $\begin{array}{c}\text { APS } \\
\text { (ESF/UBS) }\end{array}$ & \multicolumn{2}{|c|}{ SERVIÇO ESPECIALIZADO } \\
\hline \multicolumn{3}{|c|}{$\begin{array}{l}\text { Combater preconceito; } \\
\text { Conscientizar a população quanto à prevenção, promoção, ao diagnóstico e à assistência; } \\
\text { Realizar aconselhamento para testes e adesão à TARV. }\end{array}$} \\
\hline & \multicolumn{2}{|c|}{$\begin{array}{l}\text { Oferecer tratamento psicológico e nutricional; } \\
\text { Exames laboratoriais. }\end{array}$} \\
\hline \multicolumn{3}{|c|}{$\begin{array}{l}\text { Garantir acesso às populações mais vulneráveis; } \\
\text { Realizar teste rápido; } \\
\text { Promover a inserção social. }\end{array}$} \\
\hline \multicolumn{3}{|c|}{ ARTICULAÇÃO } \\
\hline \multicolumn{3}{|c|}{$\begin{array}{l}\text { Organizar atendimento por idade: >14 anos } 11 \text { meses e } 29 \text { dias no HU e <14 anos } 11 \text { meses e } 29 \text { dias no CTA; } \\
\text { Realizar transição gradual do adolescente para o CTA; } \\
\text { Estabelecer estratificação de risco para compartilhar o atendimento entre os pontos: CD4 baixo e CV alta permanecem } \\
\text { no serviço especializado, e compartilha com a APS o atendimento de puericultura/hebiatria. Para CD4 alto e carga viral } \\
\text { indetectável, o serviço especializado transferirá o atendimento para APS, com avaliação trimestral na clínica do HIV. }\end{array}$} \\
\hline \multicolumn{3}{|c|}{$\begin{array}{l}\text { Estabelecer a transferência dos usuários entre os pontos de atenção à saúde; } \\
\text { Realizar contato telefônico entre os pontos para a coordenação do cuidado; } \\
\text { Realizar busca ativa e monitoramento de crianças/adolescentes com HIV no território da APS; } \\
\text { Registrar os usuários nos pontos de atenção à saúde; } \\
\text { Estabelecer um profissional de referência nos serviços; } \\
\text { Implantar instrumento de transferência entre os pontos, com o histórico do usuário. }\end{array}$} \\
\hline \multicolumn{3}{|c|}{ VALIDAÇÃO DAS IDEIAS CENTRAIS } \\
\hline Linha de Cuidado para crianças e ad & sentes vivendo com HIV & \\
\hline
\end{tabular}

Legenda: APS = Atenção Primária a Saúde; CD4 = cluster of differentation; CTA = Centro de Testagem e Aconselhamento; CV = Carga Viral; ESF = Estratégias de Saúde da Família; HIV = vírus da imunodeficiência humana; HU = hospital universitário; IST = Infecção Sexualmente Transmissível; TARV = Terapia antirretroviral; UBS = Unidades Básicas de Saúde.

Fonte: Grupo Focal, elaboração própria.

\section{DISCUSSÃO}

A discussão da condição crônica do HIV foi pautada no aumento da sobrevida de crianças e adolescentes infectadose na ampliação dos cuidados, e a rotina de acompanhamento da infecção se somou aos cuidados habituais de desenvolvimento de modo permanente. ${ }^{8}$ No entanto, a organização do SUS, o funcionamento dos serviços e a atenção desenvolvida pelos profissionais ainda possui como foco a assistência às condições agudas de saúde.

Reconhecemos que há indicação de organização do sistema de saúde para a atenção às condições crônicas, sem deixar de acolher as outras demandas. Isto reflete, consequentemente, em desafios para os profissionais e para os serviços de saúde apoiados pela política pública como da RAS. ${ }^{14,15}$ Para tanto, é 


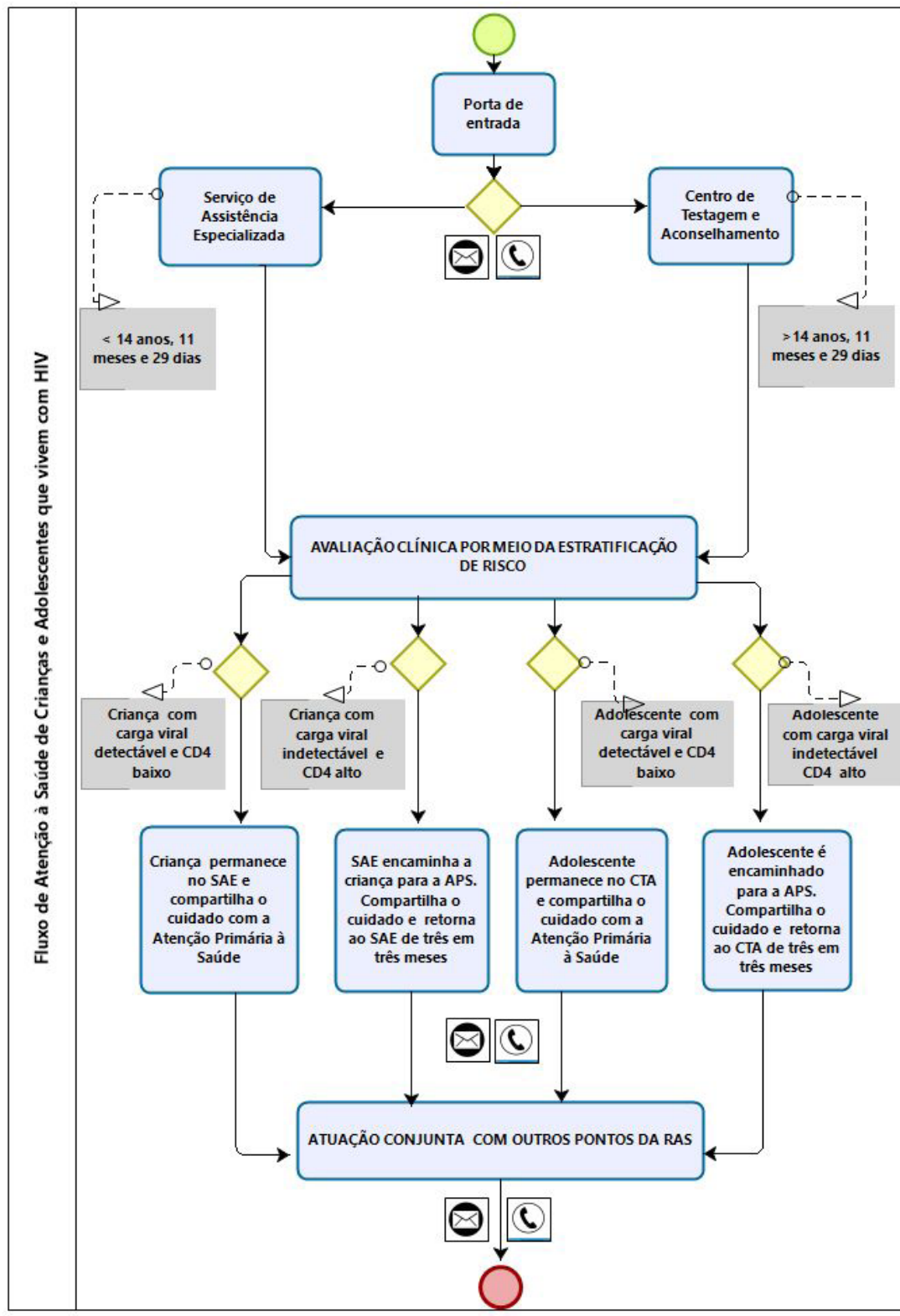

Legenda:

Início do fluxo - porta de entrada- serviços como: Atenção Primária à Saúde, Serviço de Assistência Especializada, Centro de Testagem e Aconselhamento, Hospitais, Pronto Atendimentos, Unidades de Pronto Atendimento.

Tomada de decisão pelos profissionais de saúde

Final do fluxo

Associação de informações que justificam a tomada de decisão

$\longrightarrow$ Fluxo de sequência: mostra a ordem que as atividades serão executadas

(1) 10 Encaminhamentos entre os pontos da Rede de Atenção à Saúde. A comunicação deve ser realizada por contato telefônico e por escrito, via e-mail, entre profissionais de saúde.

Figura 1. Linha de cuidado para crianças e adolescentes vivendo com HIV. Brasil, 2015.

Fonte: elaborada pelas autoras, 2020. 
primordial a promoção de educação permanente dos profissionais de saúde e de incentivo ao trabalho multidisciplinar com o devido monitoramento dos processos e dos resultados, que pode ser mediado pelo uso da tecnologia da informação.

As evidências científicas mostram como tem acontecido o acompanhamento de saúde da criança e do adolescente vivendo com HIV em serviços especializados ou hospitalares. $\mathrm{Na}$ atenção à saúde dessa população, predomina o enfoque biologicista, médico-centrado, com foco no controle clínico e no tratamento, ${ }^{7,16}$ enquanto nos serviços de APS há ênfase em aconselhamento, educação em saúde e distribuição de insumos de prevenção. Entretanto, há escassez de ações às pessoas vivendo com HIV. ${ }^{4} \mathrm{~A}$ isso se soma a barreira de uma RAS desarticulada, ${ }^{17}$ sendo assim, reconhecemos que as ações para essa população são desenvolvidas de forma fragmentada, com avanços no atendimento às urgências clínicas advindas da infecção, especialmente pelo vínculo com o serviço especializado. Este é elemento conceitual discutido entre os participantes do estudo em tela.

A fragmentação da organização da atenção à saúde intensifica a busca por serviços de maior densidade tecnológica, o que mantém o não reconhecimento da APS como fonte de primeiro contato, inclusive devido à percepção pela família da falta de preparo dos profissionais para lidar com a condição crônica e da falta de resolutividade na APS. Isto fragiliza ainda mais o vínculo, gera insatisfação e potencializa o descrédito dos serviços de APS. ${ }^{18}$

Compreendemos que o estabelecimento de vínculos entre usuários e profissionais pressupõe o acesso ao serviço de APS e um contato continuado, que resulta na confiança. Ainda, o desenvolvimento de ações resolutivas pode repercutir na manutenção da APS como fonte regular de atenção e, inclusive, reduzir a utilização de serviços especializados.

Cabe salientar que a escolha pela fonte regular de atenção se dá pela facilidade de acesso ao serviço especializado e pelo sistema ineficiente de transferência dos usuários entre os pontos da RAS. ${ }^{7}$ Entretanto, o fortalecimento do SUS implica a coordenação do fluxo dos usuários e o estabelecimento de uma corresponsabilidade entre os profissionais. Para obter ações resolutivas na assistência integral à saúde, recomendase aos serviços especializados atuarem conjuntamente com a APS $^{6,16}$ para ações de promoção, monitoramento e suporte à retenção e adesão ao tratamento. ${ }^{5,15}$

Para minimizar a fragmentação ${ }^{18}$ e caracterizar uma RAS fortalecida, ${ }^{9}$ há que reorganizar a atenção à saúde por meio de ações estratégicas que promovam o acesso e potencializem a vinculação dos usuários nos serviços de saúde. Essa reorganização deve ser operacionalizada por meio de diretrizes de acolhimento e construída de forma coletiva entre os serviços. Esse é o ponto convergente com a Política Nacional de Humanização do SUS, ${ }^{19}$ para a qual acolher significa manter a relação entre equipes e serviços a partir da análise dos processos de trabalho.

Entretanto, os profissionais dos serviços de APS, participantes desse estudo, não identificaram em seu território e não realizam atendimento algum a crianças e adolescentes que vivem com HIV. Por vezes, eles também desconhecem as ações realizadas pelo serviço especializado em HIV. Essas constituem barreiras, inclusive, para a coordenação da atenção à saúde desses usuários. Almeja-se que a cobertura seja feita por meio de ESF; todavia, nacionalmente, a distribuição entre ESF e UBS tradicionais ainda é heterogênea. ${ }^{20}$

Infere-se que os profissionais da APS não conhecem essas crianças em seu território porque as famílias não a acessam ou não revelam o diagnóstico. Concordamos com o esforço em direção à constituição de multiterritórios para a atenção a essa população, aliado à qualificação das práticas dos profissionais, com preservação do sigilo das informações e respeito aos direitos humanos. Também, entendemos ser imprescindível respeitar o ponto de acesso preferencial das famílias devido ao direito ao sigilo do diagnóstico e à proteção contra preconceito e discriminação.

A política pública, por si só, pressupõe a territorialização e adscrição dos usuários. Além disso, garante o vínculo e a facilidade de acesso à unidade de saúde pelos usuários. ${ }^{15}$ É necessário tanto empreender esforços para ampliação da cobertura de ESF quanto refletir sobre esse pressuposto, considerando os aspectos sociais e subjetivos associados à epidemia do HIV.

A RAS tem como base de seus processos a população sob sua responsabilidade, segundo suas necessidades específicas. Contudo, a proximidade territorial pode tanto auxiliar quanto dificultar o acesso, podendo haver um deslocamento para fora da comunidade local na busca de atenção à saúde. Deve-se considerar o caráter extraterritorial do vínculo, sustentado nas relações de confiança e acolhimento. Então, há possibilidade de uma perspectiva de multiterritorialidade, oferecendo diversas possibilidades de pontos de entrada para o acesso. ${ }^{21}$

Corrobora-se o rompimento da imposição do acesso ao serviço de APS, estabelecido pela territorialização segundo critério espacial. Contudo, consideramos o aspecto relacional como potencializador da longitudinalidade, pela preferência da família e com fluxo de informações, comunicações e pessoas entre os serviços para a coordenação do cuidado.

Contudo, rompendo-se com a imposição territorial, tem-se um serviço especializado superlotado. Tal condição converge para as experiências de outros municípios em situações de atendimento para $95 \%$ da população vivendo com HIV. ${ }^{22}$ Isso se dá, muitas vezes, pela exigência de recursos, como equipe de profissionais com experiência e estrutura no serviço, bem como tecnologia para o acompanhamento clínico e laboratorial. ${ }^{23}$

Há, também, indefinição da LC e de fluxos de transferência entre os serviços, ${ }^{24}$ evidenciando a necessidade de articulação entre os pontos da RAS. As transferências são consideradas insatisfatórias/insuficientes, especialmente, quanto à falta de definição de quais pontos são indicados para o encaminhamento dos resultados da consulta entre as equipes. ${ }^{25}$

Isso se reflete na avaliação insatisfatória do atributo coordenação - integração de cuidados, segundo os familiares de crianças com condição crônica. ${ }^{26}$ Aprendemos que, para 
preservar a continuidade de um cuidado em qualquer ponto de atenção, independente da densidade tecnológica, é necessária a disponibilização do prontuário, de modo eletrônico ou não, além de apoio matricial.

Os profissionais participantes do GF relataram que uma das situações contribuidoras para a manutenção de um atendimento centrado em especialidades é o limite de estrutura física e de pessoal. A estrutura física limitada é sinalizada em serviços de atendimento às pessoas vivendo com HIV, nos quais o reduzido espaço inviabiliza a privacidade e o conforto. Os serviços especializados estão sobrecarregados devido à centralidade do atendimento, bem como à carência de recursos humanos e de infraestrutura adequada. $5,26,27$

A sobrecarga dos profissionais perpassou o cenário dos serviços especializados e das equipes de ESF em razão, também, de terem que assumir a responsabilidade por mais uma área de ações, o que exige reconfiguração do processo de trabalho. Além disso, há equipes incompletas em UBS, restringindo o acesso à população. Esses aspectos dificultam o vínculo, a referência e a responsabilização do serviço sobre o território de atuação, considerados qualificadores da APS. ${ }^{6}$

Nos serviços de maior densidade tecnológica, a escassez de especialistas é uma das dificuldades para o desenvolvimento de uma atenção com base nos atributos integralidade e coordenação. ${ }^{6,28}$ Com relação aos serviços de APS, a alta rotatividade prejudica a longitudinalidade, além de exigir que mais recursos públicos sejam gastos na contratação, na capacitação e preparação dos novos profissionais. ${ }^{29}$ Ainda assim, entre os profissionais da APS há falta de um consenso sobre essa população pertencer ao seu campo de atuação. Isso reaparece em questões que, teoricamente, já deveriam ter sido superadas, tais como a implantação do teste rápido. ${ }^{30}$

A dificuldade de afiliação à APS de pessoas vivendo com HIV é justificada pela falta de preparo profissional para desenvolver a atenção a essa população e pelo estigma., ${ }^{4,2431}$ Há necessidade de sensibilização, acompanhamento e qualificação. Essas situações recorrentes demandam a educação permanente como indutora de mudanças nos processos de trabalho, de modo a corresponsabilizar os componentes dos diversos pontos da RAS ${ }^{15} \mathrm{e}$ alcançar o compartilhamento, em vez de simples transferência de responsabilidade no processo de descentralização. Isto envolve, além do compromisso do profissional, as condições estruturais para o processo ser desenvolvido. ${ }^{6}$

Entendemos que, para promover a integração dos serviços, faz-se necessário que a equipe estabeleça o objetivo do cuidado colaborativo, fortalecido pela capacidade de comunicação. Além disso, é preciso definir a atribuição das ações de cada serviço, o comprometimento por parte do governo em relação a uma política e legislação, inclusive àqueles colaboradores vindos de outros setores não relacionados com a saúde. Porém, tais perspectivas implicam o desenvolvimento de educação continuada para qualificação dos profissionais da APS.

O elemento social foi discutido pelos profissionais, constituindose no estigma do próprio usuário em relação à sua condição de saúde. Isso acarreta resistência do usuário em revelar a sua condição sorológica para o profissional de saúde. Ele não revela por medo de sua exposição na comunidade, optando, assim, por serviços especializados distantes de seu convívio social. ${ }^{32}$

As pessoas vivendo com HIV têm receio da procura por serviços próximos ao local de moradia pelo risco da difusão de dados confidenciais, de serem identificadas ou de encontrarem pessoas conhecidas..$^{22} \mathrm{O}$ desafio de ordem moral aponta a dificuldade do entendimento do HIV como uma condição crônica de saúde, ainda marcada por estigma. Soma-se o de ordem ética, relativo à importância do sigilo e da confidencialidade em um contexto de trabalho de equipe territorializada. ${ }^{4} \mathrm{~A}$ falta de confiança dos usuários nos profissionais da APS em relação ao sigilo de sua condição sorológica também é determinante para preferência pelo serviço especializado. ${ }^{33}$

O planejamento das ações de transferência, acolhimento, atendimento e comunicação entre os serviços, bem como a capacitação dos profissionais, exige o desenvolvimento de estratégias para gerir a informação do diagnóstico e as demandas de sigilo, visando promover o acesso aos serviços e a construção de vínculo e confiança entre usuários e profissionais. ${ }^{23}$

Reitera-se que a territorialidade amplia as possibilidades de cuidado e também de exposição a preconceitos. Portanto, representa o desafio de ordem organizacional interna, externa e política. A interna diz respeito à flexibilidade de a APS considerar as necessidades dos usuários para adaptar seus modos de organização, a externa remete à interação e apoio entre os médicos de família e os infectologistas, e a política se refere ao diálogo entre diferentes atores para a condução da agenda. ${ }^{4}$

Os profissionais debateram como poderia se dar a descentralização da atenção à saúde, pautada nas diretrizes de $\mathrm{LC}^{34} \mathrm{em}$ que se pretende a articulação de recursos e práticas de saúde, como de diagnóstico e terapia, entre as respostas de determinado território. A LC é uma ferramenta de ajuda na organização da RAS e se propõe a identificar os múltiplos caminhos possíveis, priorizando percursos efetivos e racionais, aproximando a gestão e o cuidado e funcionando com base nos projetos terapêuticos. A efetivação da LC tem como primeira instância a pactuação entre todos os órgãos envolvidos. ${ }^{15}$ Entendemos ser necessário que gestores e profissionais protagonizem o processo de reorganização das ações e dos serviços de saúde.

Um dos elementos constitutivos das RAS é a estrutura operacional, a qual é constituída pelos nós das redes e pelas ligações que os comunicam. Essa estrutura possui cinco componentes: 1) o centro de comunicação - a APS -; 2) os pontos de atenção secundários e terciários; 3 ) os sistemas de apoio; 4) os sistemas logísticos; e 5) o sistema de governança da RAS. Com relação aos nós das redes, estes correspondem aos três primeiros. As ligações, que comunicam os diferentes nós, correspondem ao quarto componente, e o último corresponde àquele que governa as relações entre os quatro primeiros. ${ }^{35}$

Sabe-se que serviço especializado desenvolve o cuidado específico relacionado à evolução da infecção pelo HIV com as demandas da clínica e da conduta terapêutica. No entanto, a 
coordenação da atenção e o acompanhamento do usuário na sua comunidade são atribuições da APS, e tal determinação deverá estar clara entre os serviços. Isto foi representado pelos participantes na LC (Figura 1).

Assim, a atenção à saúde na puericultura e na puberdade e o calendário de imunizações devem ser mantidos na APS, considerando a rede social e afetiva da criança e adolescente vivendo com HIV. ${ }^{10}$ Pesquisa anterior, neste mesmo município, apontou a imperativa de a integração entre os serviços ser estabelecida por meio de um fluxo de usuários no SUS. ${ }^{10}$ Entende-se como essencial a pactuação, a contratualização e a conectividade de papéis e de tarefas entre diferentes serviços e os profissionais respectivos, a partir da APS. ${ }^{15}$

\section{CONSIDERAÇÕES FINAIS E IMPLICAÇÕES PARA A PRÁTICA}

Concluímos que a abordagem participante dos profissionais e gestores possibilitou o protagonismo dos mesmos, uma vez que construíram coletivamente os resultados da pesquisa. A discussão foi profícua acerca da constituição da RAS em busca de melhores resultados na saúde das crianças e adolescentes vivendo com HIV, e culminou em um produto viável de ser implantado ao cotidiano assistencial. Mas, a implantação desse produto, denominado LC requer ações estratégicas locais, com base na discussão da realidade dos recursos, das potencialidades e fragilidades dos serviços para a integração dos pontos de atenção das RAS.

Reconhecemos a existência de desafios, mas apontamos possibilidades para a continuidade de ações que avancem no sentido da atenção em rede, e de que APS se fortaleça e possa contar com o apoio dos serviços especializados no cuidado a essas crianças, adolescentes e suas famílias. Para superar o modelo tradicional, a coordenação do cuidado precisa da corresponsabilização dos profissionais dos diferentes tipos de serviço, de modo a promover a integralidade e a continuidade das ações individuais e coletivas. A limitação da pesquisa reside na necessidade de incluir outros pontos da RAS como portas de entrada do SUS e/ou facilitadores de acesso às crianças e adolescentes vivendo com HIV.

\section{AGRADECIMENTOS}

As autoras agradecem à Dra Laura Ferreira Cortes, pelo apoio no desenvolvimento do grupo focal, como observadora do tipo participante, na etapa de campo de produção de dados da presente pesquisa.

\section{FINANCIAMENTO}

Programa de Pesquisa para o SUS e Fundação de Amparo a Pesquisa do Estado do Rio Grande do Sul (PPSUS/ FAPERGS-2013-2014): Número do Processo: 1217-2551/13-0; Conselho Nacional de Desenvolvimento Científico e Tecnológico (CNPq)- edital Universal (2013-2016): Número do Processo: 482554/2013-4; Produtividade em Pesquisa- PQ-2014. Número do Processo: 307350/2014-2. O presente trabalho foi realizado com apoio da Coordenação de Aperfeiçoamento de Pessoal de Nível Superior - Brasil (CAPES) - Código de Financiamento 001.

\section{CONTRIBUIÇÕES DOS AUTORES}

Desenho do estudo. Daniela Dal Forno Kinalski. Raquel Einloft Kleinubing. Stela Maris de Mello Padoin. Cristiane Cardoso de Paula.

Coleta ou produção dos dados. Daniela Dal Forno Kinalski. Raquel Einloft Kleinubing. Stela Maris de Mello Padoin. Cristiane Cardoso de Paula

Análise de dados. Daniela Dal Forno Kinalski. Raquel Einloft Kleinubing. Stela Maris de Mello Padoin. Cristiane Cardoso de Paula.

Interpretação dos resultados. Daniela Dal Forno Kinalski. Raquel Einloft Kleinubing. Stela Maris de Mello Padoin. Cristiane Cardoso de Paula.

Redação e revisão crítica do manuscrito. Daniela Dal Forno Kinalski. Raquel Einloft Kleinubing. Marília Alessandra Bick. Tassiane Ferreira Langendorf. Stela Maris de Mello Padoin. Cristiane Cardoso de Paula.

Aprovação da versão final do artigo. Daniela Dal Forno Kinalski. Raquel Einloft Kleinubing. Marília Alessandra Bick. Tassiane Ferreira Langendorf. Stela Maris de Mello Padoin. Cristiane Cardoso de Paula.

Responsabilidade por todos os aspectos do conteúdo e a integridade do artigo publicado. Daniela Dal Forno Kinalski. Raquel Einloft Kleinubing. Marília Alessandra Bick. Tassiane Ferreira Langendorf. Stela Maris de Mello Padoin. Cristiane Cardoso de Paula.

\section{EDITOR ASSOCIADO}

\author{
Aline Cristiane Okido
}

\section{REFERÊNCIAS}

1. United Nations. Special edition: progress towards the Sustainable Development Goals. New York: United Nations; 2019.

2. World Health Organization, Pan American Health Organization. Plan of action for the prevention and control of HIV and sexually transmitted infections 2016-2021. Geneva: WHO; 2016.

3. The Joint United Nations Programme on HIV/AIDS. On the Fast-Track to end AIDS free generation. Geneva: UNAIDS; 2015.

4. Melo EA, Maksud I, Agostini R. Cuidado, HIV/Aids e atenção primária no Brasil: desafio para a atenção no Sistema Único de Saúde? Rev Panam Salud Publica. 2018 set;42:e151. http://dx.doi.org/10.26633/ RPSP.2018.151. PMid:31093179.

5. Loch AP, Nemes MIB, Santos MA, Alves AM, Melchior R, Basso $\mathrm{CR}$ et al. Avaliação dos serviços ambulatoriais de assistência a pessoas vivendo com HIV no Sistema Único de Saúde: estudo comparativo 2007/2010. Cad Saude Publica. 2018;34(2):e00047217. http://dx.doi. org/10.1590/0102-311x00047217. PMid:29489944

6. Zambenedetti G, Silva RAN. Descentralização da atenção em HIV-Aids para a atenção básica: tensões e potencialidades. Physis. 2016 jul/ set;26(3):785-806. http://dx.doi.org/10.1590/s0103-73312016000300005.

7. Magnabosco GT, Lopes LM, Andrade RLP, Brunello MEF, Monroe AA, Villa TCS. HIV/AIDS care: analysis of actions and health services 
integration. Esc Anna Nery. 2018 jul;22(4):e20180015. http://dx.doi. org/10.1590/2177-9465-ean-2018-0015.

8. Sohn AH. Taking a critical look at the UNAIDS global estimates on pediatric and adolescent HIV survival and death. J Int AIDS Soc. 2017 jun;20(1). http://dx.doi.org/10.7448/IAS.20.1.21952.

9. Nakata LC, Feltrin AFS, Chaves LDP, Ferreira JBB. Concept of health care network and its key characteristics: a scoping review. Esc Anna Nery. 2020 jan;24(2):e20190154. http://dx.doi.org/10.1590/2177-9465ean-2019-0154.

10. Silva CB, Paula CC, Lopes LFD, Harzheim E, Magnago TSBS, Schimith MD. Atenção à saúde de criança e adolescente com HIV: comparação entre serviços. Rev Bras Enferm. 2016 maio/jun;69(3):522-31. http:// dx.doi.org/10.1590/0034-7167.2016690315i. PMid:27355302.

11. Grieb SD, Eder MM, Smith KC, Calhoun K, Tandon D. Qualitative research and community-based participatory research: considerations for effective dissemination in the peer-reviewed literature. Prog Community Health Partnersh. 2015 dez;9(2):275-82. http://dx.doi.org/10.1353/ cpr.2015.0041. PMid:26412768.

12. Kinalski DDF, Paula CC, Padoin SMM, Neves ET, Kleinubing RE, Cortes LF. Focus group on qualitative research: experience report. Rev Bras Enferm. 2017;70(2):424-9. http://dx.doi.org/10.1590/0034-7167-20160091. PMid:28403311.

13. Minayo MCS. O desafio do conhecimento: pesquisa qualitativa em saúde. 14. ed. São Paulo: Hucitec/ABRASCO; 2014.

14. Portaria $\mathrm{n}^{\circ} 4.279$, de 30 de dezembro de 2010 (BR). Estabelece diretrizes para a organização da Rede de Atenção à Saúde no âmbito do Sistema Único de Saúde (SUS). Diário Oficial da União [periódico na internet], Brasília (DF), 31 dez 2010 [citado 10 jul 2020]. Disponível em: https:// www.jusbrasil.com.br/diarios/24023258/pg-88-secao-1-diario-oficialda-uniao-dou-de-31-12-2010

15. Rio Grande do Sul, Secretaria do Estado da Saúde, Departamento de Ações em Saúde, Coordenação Estadual de IST/AIDS. Linha de cuidado para pessoas vivendo com HIV (PVHIV) e outras infecções sexualmente transmissíveis. 2. ed. Porto Alegre (RS): Escola de Saúde Pública; 2018.

16. Medeiros LB, Trigueiro DRSG, Silva DM, Nascimento JA, Monroe AA, Nogueira JÁ et al. Integração entre serviços de saúde no cuidado às pessoas vivendo com Aids: uma abordagem utilizando árvore de decisão. Cien Saude Colet. 2016 fev;21(2):543-52. http://dx.doi. org/10.1590/1413-81232015212.06102015. PMid:26910161.

17. Viana ALÁ, Bousquat A, Melo GA, Negri Fo A, Medina MG. Regionalização e Redes de Saúde. Cien Saude Colet. 2018 jun;23(6):1791-8. http:// dx.doi.org/10.1590/1413-81232018236.05502018. PMid:29972487.

18. Silva MEA, Reichert APS, Souza SAF, Pimenta EAG, Collet N. Doença crônica na infância e adolescência: vínculos da família na rede de atenção à saúde. Texto Contexto Enferm. 2018 maio;27(2):e4460016. http://dx.doi.org/10.1590/0104-070720180004460016.

19. Ministério da Saúde (BR), Secretaria de Atenção à Saúde. Núcleo Técnico da Política Nacional de Humanização. Humaniza SUS: documento base para gestores e trabalhadores do SUS. Brasília (DF): Ministério da Saúde; 2006.

20. Neves RG, Flores TR, Duro SMS, Nunes BP, Tomasi E. Time trend of Family Health Strategy coverage in Brazil, its Regions and Federative Units, 2006-2016. Epidemiol Serv Saude. 2018 set;27(3):e2017170. http://dx.doi.org/10.5123/s1679-49742018000300008. PMid:30183868.

21. Zambenedetti G, Silva RAN. O paradoxo do território e os processos de estigmatização no acesso ao diagnóstico de HIV na atenção básica em saúde. Estud Psicol. 2015 out/dez;20(4). http://dx.doi.org/10.5935/16784669.20150024.
22. Affeldt AB, Silveira MF, Barcelos RS. Perfil de pessoas idosas vivendo com HIV/aids em Pelotas, sul do Brasil, 1998 a 2013. Epidemiol Serv Saude. 2015;24(1):79-86. http://dx.doi.org/10.5123/S167949742015000100009.

23. Astolfo S, Kehrig RT, Oliveira LR. Disponibilidade de recursos dos serviços ambulatoriais do Sistema Único de Saúde destinados a pessoas vivendo com HIV em Mato Grosso, 2016. Epidemiol Serv Saude. 2018;27(3):e2017406. http://dx.doi.org/10.5123/S167949742018000300001. PMid:30365700.

24. Colaço AD, Meirelles BHS, Heidemann ITSB, Villarinho MV. Care for the person who lives with HIV/AIDS in primary health care. Texto Contexto Enferm. 2019 jun;28:e20170339. http://dx.doi.org/10.1590/1980-265xtce-2017-0339.

25. Kinalski DDF, Oliveira APS, Paula CC, Padoin SMM, Schmidt MD, Kleinubing RE. Factores que interfieren en el atributo coordinación en la atención primaria de la salud: revisión integrativa. Enfermería Comunitaria [Internet]. 2018; [citado 2020 maio 22];14. Disponível em: http://ciberindex.com/c/ec/e11079

26. Wolkers PCB, Macedo JCB, Rodrigues CM, Furtado MCC, Mello DF. Primary care for children with type 1 diabetes mellitus: caregiver perspectives. Acta Paul Enferm. 2017;30(5):451-7. http://dx.doi. org/10.1590/1982-0194201700066.

27. Carvalho VKA, Godoi DF, Perini FB, Vidor AC. Cuidado compartilhado de pessoas vivendo com HIV/AIDS na Atenção Primária: resultados da descentralização em Florianópolis. Rev Bras Med Fam Com. 2020;15(42):2066. http://dx.doi.org/10.5712/rbmfc15(42)2066.

28. Monteiro S, Brigeiro M. Prevenção do HIV/Aids em municípios da Baixada Fluminense, Rio de Janeiro, Brasil: hiatos entre a política global atual e as respostas locais. Interface. 2019;23:e180410. http:// dx.doi.org/10.1590/interface.180410.

29. Vaz EMC, Collet N, Cursino EG, Forte FDS, Magalhães RKBP, Reichert APS. Coordenação do cuidado na Atenção à Saúde à(ao) criança/ adolescente em condição crônica. Rev Bras Enferm. 2018;71(Supl 6):26129. http://dx.doi.org/10.1590/0034-7167-2017-0787. PMid:30540035.

30. Ew RAS, Ferreira GS, Moro LM, Rocha KB. Estigma e teste rápido na atenção básica: percepção de usuários e profissionais. Rev Bras Promoç Saúde. 2018;31(3). http://dx.doi.org/10.5020/18061230.2018.7463.

31. Junges JR, Recktenwald M, Herbert NDR, Moretti AW, Tomasini F, Pereira BNK. Sigilo e privacidade das informações sobre usuário nas equipes de atenção básica à saúde: revisão. Rev Bioet. 2015 abr;23(1):200-6. http://dx.doi.org/10.1590/1983-80422015231060.

32. Navarro AMA, Bezerra VP, Oliveira DA, Moreira MAS, Alves MSCF, Gurge SN. Representações sociais do HIV/Aids: percepção dos profissionais da atenção primária à saúde. R Pesq Cuid Fundam [Internet]. 2011 dez; [citado 2020 maio 22];(Supl). Disponível em: http://www.seer.unirio.br/ index.php/cuidadofundamental/article/view/1966/pdf_529

33. Dantas MS, Abrão FMS, Costa SFG, Oliveira DC. HIV/AIDS: meanings given by male health professionals. Esc Anna Nery. 2015 abr/jun;19(2). http://dx.doi.org/10.5935/1414-8145.20150044.

34. Resolução №663/2014-CIB/RS (RS). Proposta de Linha de Cuidado para Pessoas Vivendo com HIV/Aids (PVHA) e outras DST.Diário Oficia do Rio Grande do Sul [periódico na internet], Porto Alegre (RS), 11 nov 2014 [citado 26 maio 2020]. Disponível em: https://saude.rs.gov.br/ upload/arquivos/carga20170208/23110836-1419963216-cibr663-14. pdf

35. Mendes EV. As redes de atenção à saúde. Cien Saude Colet. 2010;15(5):2297-305. http://dx.doi.org/10.1590/S1413-81232010000500005. PMid:20802863.

artigo extraído da dissertação de mestrado de autoria de Daniela Dal Forno Kinalski, defendida no ano de 2016, Programa de Pós-graduação em Enfermagem Universidade Federal de Santa Maria, sob a orientação de Cristiane Cardoso de Paula. Texto completo disponível em: http://coral.ufsm.br/ppgent/images/ Dissertacoes_alunos/Dissertacao_Daniela_Dal_Forno_Kinalski.pdf 